\title{
Simulation d'étirement d'impulsions brèves
}

\author{
J.C. Venturino \\ Optis, ZE La Farlède, rue Parmentier, BP. 275, 83078 Toulon cedex 9, France
}

\begin{abstract}
Résumé : Cette simulation d'impulsions brèves a été réalisée dans Solstis. Ce logiciel est destiné à modéliser la propagation lumineuse à travers des systèmes optiques dont la propagation laser. Ainsi, on peut étudier l'effet de l'étirement d'impulsions brèves dans un système classique et voir les effets d'aberrations sur cet étirement.
\end{abstract}

\section{BUT DE LA MODELISATION}

Le but de cette modélisation est d'une part, de pouvoir étudier le comportement d'une impulsion brève dans un grand nombre de cas facilement mis en œuvre et d'autre part, de pouvoir étudier simultanément l'influence des aberrations de tout types (sphérique, chromatique, ...) sur la durée d'impulsion.

En effet, plusieurs calculs théoriques existent pour modéliser le comportement d'une impulsion brève lors de la propagation aussi bien au niveau de l'élargissement lié à la dispersion que du retard introduit entre le centre du champ et le bord de champ. Des calculs existent également pour simuler l'effet d'aberrations dans des cas simples. La simulation numérique de cette propagation permet alors de pouvoir tenir compte des effets de dispersion au niveau spatial (aberration chromatique) ainsi que ceux des aberrations géométriques du système étudié.

Plusieurs séries de validations ont été réalisées pour retrouver les résultats calculés théoriquement dans des cas simples aussi bien au niveau de l'élargissement après propagation qu'au niveau du retard introduit dans le champ.

\section{PRINCIPE DE FONCTIONNEMENT}

Deux types de propagation sont possibles : la propagation géométrique et la propagation cohérente.

\subsection{Propagation géométrique}

Le principe de cette partie repose sur la propagation d'un rayon sur l'axe optique pour chaque longueur d'onde du spectre de l'impulsion. A partir du spectre déterminé en entrée du système et du chemin optique calculé pendant la propagation pour chaque longueur d'onde, on peut calculer la phase, le profil spectral et temporel en sortie du système.

$$
\varphi(\omega)=\varphi\left(\omega_{0}\right)+\left(\frac{d \varphi}{d \omega}\right)\left(\omega-\omega_{0}\right)_{\omega_{0}}+\left(\frac{d^{2} \varphi}{d \omega^{2}}\right) \frac{\left(\omega-\omega_{0}\right)^{2} \omega_{0}}{2}+\left(\frac{d^{3} \varphi}{d \omega^{3}}\right) \frac{\left(\omega-\omega_{0}\right)^{3} \omega_{0}}{6}+\cdots
$$

\subsection{Propagation cohérente}

Le principe de cette partie repose sur la propagation cohérente et le calcul de carte d'éclairement pour chaque longueur d'onde du spectre de l'impulsion. A partir du spectre déterminé en entrée du système et des différentes cartes calculées à chaque longueur d'onde, on peut calculer le profil spatial intégré temporellement en ajoutant toutes les cartes. On calcule également le profil temporel sur un pixel de la carte finale ou le profil temporel intégré spatiallement. 


$$
F(x, y)=\sum_{\lambda_{i}} A_{\lambda_{i}}(x, y) \cdot A_{\lambda_{i}}^{*}(x, y) \cdot \frac{W_{\lambda_{i}}}{\sum_{x} \sum_{y} A_{\lambda_{i}}(x, y) \cdot A_{\lambda_{i}}^{*}(x, y)} \cdot \frac{N^{2}}{\Delta x \Delta y}
$$

Puisque l'on dispose de l'amplitude complexe $A$ pour chacune des longueurs d'onde $\lambda$ en chaque point de la carte, on peut alors recalculer le profil spectral et temporel de l'impulsion après la propagation. La somme de tous les profils temporels sur chacun des $\mathrm{N}$ pixels de la carte permet de calculer l'élargissement temporel après la propagation réelle. Des phénomènes comme les aberrations sont alors pris en compte.

\section{VALIDATION}

Il est possible de calculer théoriquement l'élargissement introduit lors de la propagation d'une impulsion à travers un système optique. Pour un système afocal, Bor [1] a établi la relation suivante :

$$
\begin{gathered}
\Delta T(r)=\frac{\left(r_{0}^{2}-r^{2}\right)}{2 c f_{2}(n-1)}\left(-\lambda \frac{d n}{d \lambda}\right)\left(1+\frac{1}{M}\right) \\
\text { avec } M=f_{2} / f_{1}
\end{gathered}
$$

L'avantage de ce système réside, comme l'indique la formule ci-dessus, dans la relation directe entre la position des rayons en entrée du système et l'élargissement induit en sortie. Ainsi après avoir propagé une onde plane à travers le système, on peut calculer pour plusieurs points de la carte l'élargissement par rapport au centre de la carte.

On calcule alors l'élargissement pour plusieurs position par rapport au centre de la carte.

\begin{tabular}{|c|c|c|}
\hline $\begin{array}{c}\text { Position sur la } \\
\text { lentille (mm) }\end{array}$ & $\begin{array}{c}\text { retard thé orique } \\
\text { (fs) }\end{array}$ & $\begin{array}{c}\text { Moyenne } \\
\text { Solstis (fs) }\end{array}$ \\
\hline 0 & 0 & 0 \\
\hline 5 & 1.744 & 2.5 \\
\hline 10 & 6.977 & 7.235 \\
\hline 15 & 15.7 & 16.4 \\
\hline 20 & 27.91 & 29.85 \\
\hline
\end{tabular}

Figure 1: Comparaison de validation

L'élargissement calculé par Solstis est un peu supérieur à l'élargissement théorique. Pour vérifier si cet écart est lié à la présence d'aberrations dans le système, une prochaine validation consistera à retrouver les résultats de Kempe et Rudolph [2] qui ont établi des relations permettant de calculer la durée d'impulsion dans le cas d'une lentille simple présentant de l'aberration sphérique. Un premier calcul dans a permis de mettre en évidence la différence de durée d'impulsion dans le cas où l'on se place au foyer paraxial ou au foyer marginal.

\section{Remerciements}

L'auteur tient à remercier tous ceux qui ont contribué de près ou de loin à la rédaction de cet article.

\section{Références}

1. Z.Bor. in Journal of Modern Optics, 1988, vol. 35, no 12, 1907-1918.

2. M. Kempe . and W.Rudolph in Optics Letters August 17, 1992. 